\title{
Câncer de próstata em trabalhadores rurais expostos a agrotóxicos: revisão de escopo
}

\section{Prostate cancer in rural workers exposed to agrochemicals: scope review}

\author{
Jéssica Cristina Ruths ${ }^{1}$, Sonia Mara de Andrade ${ }^{2}$, \\ Jefferson Andronio Ramundo Staduto ${ }^{3}$, Crislaine Colla ${ }^{4}$
}

\begin{abstract}
Resumo
Desde o início dos anos 2000 é crescente o número de estudos que avaliam a exposição a agrotóxicos e suas consequências para a saúde do trabalhador. Contudo, não há sistematização da produção relacionada ao câncer de próstata em trabalhadores rurais. Assim, objetivou-se mapear a literatura que trata da associação entre a exposição aos agrotóxicos e a ocorrência de câncer de próstata em trabalhadores rurais. Foi realizada uma revisão de escopo nas bases de dados Web of Science (WoS), Scopus, Cumulative Index to Nursing and Allied Health Literature (CINHAL) e Biblioteca Virtual em Saúde (BVS), empregando os descritores "Agrochemicals", "Farmers" e "Prostatic Neoplasms". Dezenove artigos fizeram parte desta revisão. Em dezessete, verificou-se associações entre a ocorrência de câncer de próstata em trabalhadores rurais e a exposição a agrotóxicos. Os fatores de risco relacionados foram: histórico familiar, tabagismo, raça/cor, idade avançada, alterações genéticas, toneladas de soja produzidas e o uso de agrotóxicos específicos. A literatura aponta a existência de consistência e plausibilidade biológica para a ocorrência de câncer de próstata em indivíduos expostos a agrotóxicos que exercem atividades agrícolas.
\end{abstract}

Palavras-chave: Trabalhadores rurais; Agroquímicos; Neoplasias da próstata.

\begin{abstract}
Since the early 2000s there has been an increasing number of studies evaluating pesticide exposure and its consequences for worker health. However, they are not systematized in relation to prostate cancer in rural workers. We aimed to map the literature dealing with the association between exposure to pesticides and the occurrence of prostate cancer in rural workers. A scoping review was conducted in the Web of Science (WoS), Scopus, Cumulative Index to Nursing and Allied Health
\end{abstract}

\footnotetext{
${ }^{1}$ Doutoranda em Desenvolvimento Regional e Agronegócio na Universidade Estadual do Oeste do Paraná (Unioeste), Toledo, Paraná, Brasil. Professora de Saúde Coletiva na Universidade Federal do Paraná (UFPR), Campus Toledo, Paraná, Brasil. E-mail: ruthsjessica@gmail.com

${ }^{2}$ Mestrado em Saúde Coletiva pela Faculdade de Odontologia de Piracicaba (FOP) da Universidade Estadual de Campinas (Unicamp), Piracicaba, São Paulo, Brasil. Docente no Curso de Medicina da Universidade Federal do Paraná, Campus Toledo, Paraná, Brasil.

${ }^{3}$ Doutorado em Ciências (Economia Aplicada) pela Universidade de São Paulo (USP), São Paulo, São Paulo, Brasil. Professor Associado do Centro de Ciências Sociais Aplicadas da Universidade Estadual do Oeste do Paraná, Toledo, Paraná, Brasil.

${ }^{4}$ Doutorado em Demografia pelo Centro de Desenvolvimento e Planejamento Regional (Cedeplar) da Universidade Federal de Minas Gerais (UFMG), Belo Horizonte, Minas Gerais, Brasil. Professora Adjunta no Curso de Ciências Econômicas da Universidade Estadual do Oeste do Paraná, Toledo, Paraná, Brasil.
} 
Literature (CINHAL) and Virtual Health Library (VHL) databases. The descriptors "Agrochemicals", "Farmers" and "Prostatic Neoplasms" were employed. Nineteen articles were part of this review. In seventeen articles, associations were found between the occurrence of prostate cancer in rural workers and exposure to agrochemicals. The related risk factors were: family history, smoking, race/ color, advanced age, genetic alterations, tons of soybeans produced, and the use of specific pesticides. The literature points to the existence of consistency and biological plausibility for the occurrence of prostate cancer in individuals exposed to pesticides who perform agricultural activities.

Keywords: Rural workers; Agrochemicals; Prostate neoplasms.

\section{Introdução}

No ano de 2018 o câncer de próstata foi o segundo mais incidente na população masculina: contabilizou-se 1,3 milhão de acometidos em todo o mundo. ${ }^{(1)}$ No Brasil, estimam-se aproximadamente 65 mil casos novos em cada ano do triênio 2020-2022; esse cenário corresponde a um risco estimado de 62,95 ocorrências para cada 100 mil homens. ${ }^{(2)}$

Apesar da importância epidemiológica do câncer de próstata e da extensa pesquisa sobre os fatores de risco envolvidos no seu processo de carcinogênese, ainda pouco se conhece sobre as suas causas. $^{(3,4)}$ A genética, idade e história familiar são fatores bem estabelecidos; encontram-se em investigação o tabagismo, excesso de gordura corporal e fatores hormonais, ambientais e ocupacionais. ${ }^{(4-7)}$

Entre os fatores ambientais e ocupacionais, vem sendo observadas associações entre a exposição a agrotóxicos e a ocorrência de câncer de próstata. ${ }^{(6,8-10)}$ Estas substâncias são manipuladas na agricultura, na pecuária, no controle de vetores e em indústrias, podendo expor os indivíduos por meio de diversas rotas de absorção (dérmica, inalação, ingestão). ${ }^{(1)}$ Impactam a saúde humana, em especial dos trabalhadores rurais, devido à sua toxicidade intrínseca, produzindo efeitos que variam conforme a forma de exposição, o princípio ativo e a dose absorvida. ${ }^{(10)}$

Desde o início dos anos 2000 é crescente o número de estudos que avaliam a exposição a agrotóxicos e suas consequências para a saúde do trabalhador. ${ }^{(5,8,12-14)}$ Há evidências de que os agrotóxicos podem agir como disruptores endócrinos, ocasionando distúrbios relacionados à infertilidade masculina, reprodução humana, anormalidades do desenvolvimento sexual e o surgimento de tumores hormônio-dependentes. ${ }^{(8,9,12)}$ Todavia, ainda não estão sistematizados estudos sobre câncer de próstata em trabalhadores rurais. Portanto, o objetivo deste estudo foi mapear a literatura que trata da associação entre a exposição aos agrotóxicos e a ocorrência de câncer de próstata em agricultores, através de uma revisão de escopo.

Em função da incidência do câncer de próstata, da magnitude das exposições e da falta de sistematização de dados sobre o tema, as reflexões deste artigo contribuíram para enriquecer o debate sobre o risco de ocorrência de câncer de próstata em trabalhadores rurais expostos a estas substâncias.

\section{Material e Método}

Trata-se de pesquisa de abordagem qualitativa, empregando a revisão de escopo (scoping review) como artifício metodológico, conforme a Preferred Reporting Items for Systematic reviews and Meta-Analyses extension for Scoping Reviews (PRISMA-ScR). A revisão de escopo é diferente das demais revisões, porque não avalia a qualidade metodológica da evidência; tem por intuito buscas exploratórias e descritivas que identifiquem lacunas de conhecimento na área estudada. ${ }^{(15)}$

Constituem etapas deste estudo: (a) elaboração da pergunta de pesquisa; (b) identificação dos estudos relevantes; (c) seleção dos estudos; (d) mapeamento dos dados; e (e) agrupamento, resumo e relação dos resultados. ${ }^{(15)}$

A estratégia População $(P)$, Conceito $(\mathrm{C})$ e Contexto (C) - (PCC) $)^{(15)}$ foi empregada para a concepção da questão norteadora deste estudo. Definiram-se: P - Agricultores; C - Exposição a agrotóxicos; e C - Neoplasia de próstata. Assim, a 
pergunta de pesquisa é: "existe associação entre a exposição de trabalhadores rurais a agrotóxicos e a ocorrência de câncer de próstata?".

O levantamento de trabalhos nas bases de dados foi executado por dois pesquisadores independentes, sem contenções no tempo de publicação e de espaço. Dúvidas e controvérsias foram analisadas e reavaliadas por todos os autores. Realizaramse buscas nas plataformas Web of Science (WoS), Scopus, Cumulative Index to Nursing and Allied Health Literature (CINHAL) e Biblioteca Virtual em Saúde (BVS), em fevereiro de 2020.

Os descritores utilizados foram escolhidos nos Descritores em Ciências da Saúde (DeCS); tendo como base a questão norteadora da pesquisa, empregaram-se "Agrochemicals", "Farmers" e "Prostatic Neoplasms"; cruzamos entre si com o uso dos booleanos "AND" e "OR", todos inseridos na língua inglesa.

Aplicou-se na avaliação dos artigos os seguintes critérios de inclusão: responder à questão norteadora desta pesquisa, estar disponível na íntegra nas bases de dados selecionadas, em acesso aberto, escritos em inglês, português ou espanhol, e de abordagem qualitativa e quantitativa. Foram excluídos artigos que não responderam à questão norteadora, que estavam em duplicidade nas bases de dados, revisões sistemáticas, de escopo, metanálises, artigos de opinião, reflexão teórica, livros, guideline, teses e dissertações.

Inicialmente, procederam-se as avaliações dos títulos, resumos e descritores; após esta etapa ocorreu a leitura na íntegra dos artigos eleitos. Os dados foram obtidos através de ferramenta de coleta de dados para revisão integrativa adaptada de Marziale. ${ }^{(16)}$

\section{Resultados}

A busca inicial nas bases de dados resultou na identificação de 264 artigos. Após as leituras de títulos e resumos selecionaram-se 94 trabalhos para leitura na íntegra. Foram excluídos 50 estudos devido à duplicidade nas bases de dados, três por não estarem disponíveis em acesso aberto, sete por se tratarem de revisões sistemáticas e metanálises e 15 em virtude de não responderem à questão norteadora desta pesquisa, ou seja, por abordar outras populações, localizações primárias de câncer ou pela ausência de tratamento da relação entre a exposição a agrotóxicos e a ocorrência do câncer de próstata.

A amostra final totalizou em 19 artigos. O procedimento de busca e seleção é apresentado na Figura 1, conforme recomendações do Joanna Briggs Institute (JBI), segundo checklist adaptado do PRISMA-ScR. ${ }^{(15)}$

Figura 1 - Fluxograma do procedimento de seleção de artigos, adaptado do PRISMA-ScR.

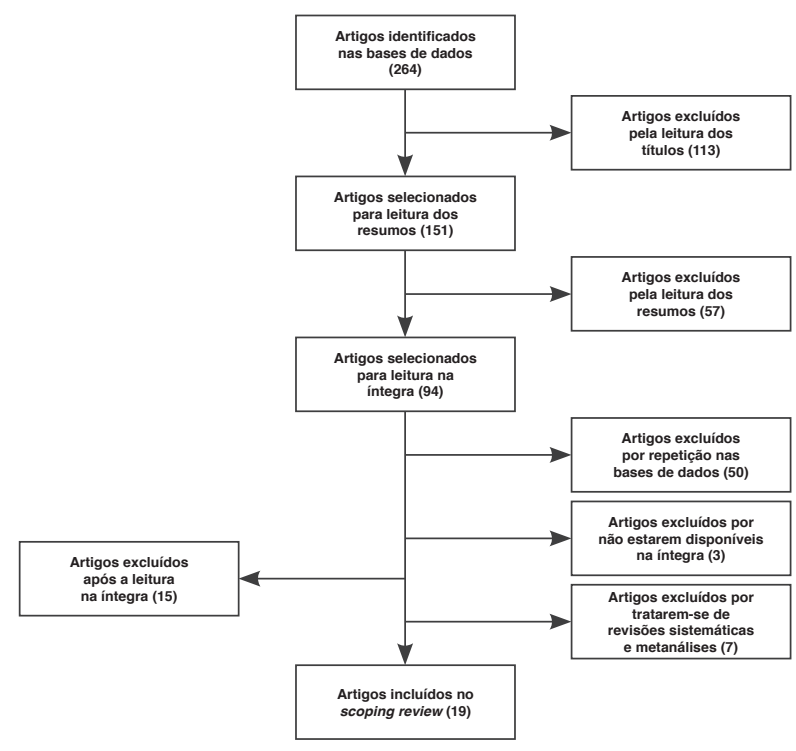

Fonte: os autores, 2020.

$\mathrm{O}$ ano de publicação dos artigos variou entre $1993^{(17)}$ e 2016. ${ }^{(8)}$ Quanto ao país de origem, 13 são dos Estados Unidos da América (EUA) ${ }^{(5,18-29)}$ três do Canadá, ${ }^{(8,17,30)}$ um da Suécia, ${ }^{(31)}$ um da Itália ${ }^{(32)}$ e um do Brasil. ${ }^{(9)}$ Apenas o artigo de Silva et al.${ }^{(9)}$ foi publicado em português, os demais encontram-se em inglês. Dez estudos fizeram parte do Agricultural Health Study (AHS). ${ }^{(19,21-22,24-29)}$

Em relação ao tipo de estudo, 11 são estudos de caso-controle, ${ }^{(8,19-20,23,26-30,32)}$ cinco são de coorte prospectiva, ${ }^{(21-22,24-25,31)}$ dois são de coorte retrospectiva $^{(17-18)}$ e um estudo ecológico. ${ }^{(9)}$ Os trabalhos discutem ocorrência do câncer de próstata relacionada com atividades agrícolas e agrotóxicos, de acordo com itens caracterizados no Quadro 1. 
Quadro 1 - Caracterização dos estudos incluídos na revisão de acordo com o autor principal, ano de publicação, local do estudo, tipo do estudo, resposta à questão norteadora da pesquisa e limitação.

\begin{tabular}{|c|c|c|c|}
\hline $\begin{array}{l}\text { Autor principal, ano de } \\
\text { publicação, local } \\
\text { e tipo do estudo. }\end{array}$ & Objetivo & Resposta à pergunta de pesquisa & Limitações \\
\hline $\begin{array}{l}\text { Parker et al., } 1999,{ }^{(18)} \text { EUA: Iowa, } \\
\text { estudo de coorte retrospectiva. }\end{array}$ & $\begin{array}{l}\left.\text { Avaliar o risco relativo ( }{ }^{\mathrm{a} R R}\right) \text { de ocorrência } \\
\text { de câncer de próstata em agricultores do } \\
\text { estado de Iowa entre } 1986 \text { e } 1989 \text {. }\end{array}$ & $\begin{array}{l}\text { Agricultores com mais de } 70 \text { anos apresentaram } \\
\text { aumento no risco de desenvolver câncer de próstata } \\
(\mathrm{RR}=2,2 ; \text { ' } \mathrm{IC} 95 \%=1,1-4,3) \text {. }\end{array}$ & $\begin{array}{l}\text { Não foi analisado quantitativamente a } \\
\text { exposições a agrotóxicos específicos. }\end{array}$ \\
\hline $\begin{array}{l}\text { Lynch et al., 2009, }{ }^{(24)} \text { EUA: Iowa } \\
\text { e Carolina do Norte, estudo de } \\
\text { coorte prospectiva. }\end{array}$ & $\begin{array}{l}\text { Analisar ocorrência de câncer de próstata } \\
\text { em aplicadores de agrotóxicos e em outras } \\
\text { categorias profissionais relacionadas, para } \\
\text { determinar uma possível relação com a } \\
\text { exposição a agrotóxicos. }\end{array}$ & $\begin{array}{l}\text { Risco significativamente elevado entre aplicadores } \\
\text { com classificação de baixa exposição a butilato } \\
(\mathrm{RR}=2,09 ; \mathrm{IC} 95 \%=1,27-3,44) \text {. No mesmo grupo } \\
\text { observou-se articulação significativamente elevada } \\
\text { do efeito da história familiar de câncer de próstata } \\
(\mathrm{RR}=2,00 ; \mathrm{IC} 95 \%=1,07-3,74) .\end{array}$ & $\begin{array}{l}\text { Número reduzido de casos expostos ao } \\
\text { butilato. Viés de recall para exposição } \\
\text { a agrotóxicos específicos. }\end{array}$ \\
\hline $\begin{array}{l}\text { Lynch et al., 2006, }{ }^{(21)} \text { EUA: Iowa } \\
\text { e Carolina do Norte, estudo de } \\
\text { coorte prospectiva. }\end{array}$ & $\begin{array}{l}\text { Avaliar a incidência de câncer de próstata } \\
\text { em aplicadores de agrotóxicos inclusos no } \\
\text { AHS expostos à cianazina. }\end{array}$ & $\begin{array}{l}\text { Não foram encontradas associações claras e } \\
\text { consistentes entre a exposição à cianazina e o } \\
\text { câncer de próstata }(\mathrm{RR}=1,23 ; \mathrm{IC} 95 \%=0,87-1,70) \text {. }\end{array}$ & $\begin{array}{l}\text { Número reduzido de casos expostos à } \\
\text { cianazina. Viés de recall para exposição } \\
\text { a agrotóxicos específicos. }\end{array}$ \\
\hline $\begin{array}{l}\text { Christensen et al., 2010, }{ }^{(25)} \text { EUA: } \\
\text { Iowa e Carolina do Norte, estudo } \\
\text { de coorte prospectiva. }\end{array}$ & $\begin{array}{l}\text { Determinar a associação entre coumafós } \\
\text { e a ocorrência de câncer de próstata } \\
\text { e comparar com a incidência entre } \\
\text { agricultores com histórico familiar } \\
\text { da doença, encontrados pelo AHS, } \\
\text { em 1993-1999 e 2000-2005. }\end{array}$ & $\begin{array}{l}\text { Em homens com histórico de câncer de próstata foi } \\
\text { observado associação estatisticamente significativa } \\
\text { entre o uso de coumafós e a ocorrência de câncer } \\
\text { de próstata }(\mathrm{RR}=1,54 ; \text { IC } 95 \%=1,13-2,38) \text {. }\end{array}$ & $\begin{array}{l}\text { Número reduzido de casos expostos ao } \\
\text { coumafós. Viés de recall para exposição } \\
\text { a agrotóxicos específicos. }\end{array}$ \\
\hline $\begin{array}{l}\text { Mahajan et al., 2006, }{ }^{(22)} \text { EUA: } \\
\text { Iowa e Carolina do Norte, } \\
\text { estudo de coorte prospectiva. }\end{array}$ & $\begin{array}{l}\text { Estudar a relação da exposição ao fonofos } \\
\text { e a incidência de câncer de próstata. }\end{array}$ & $\begin{array}{l}\text { O risco de câncer de próstata não foi vinculado ao uso } \\
\text { geral de fonofos entre os aplicadores com histórico } \\
\text { familiar da doença; porém, observou-se significativa } \\
\text { tendência à dose resposta ao longo da vida segundo os } \\
\text { dias de exposição }(\mathrm{RR}=1,77 \text {; IC } 95 \%=1,03-3,05) \text {. }\end{array}$ & $\begin{array}{l}\text { Número reduzido de casos expostos ao } \\
\text { fonofos. Viés de recall para exposição } \\
\text { a agrotóxicos específicos. }\end{array}$ \\
\hline $\begin{array}{l}\text { Morrison et al., } 1993,{ }^{(17)} \text { Canadá: } \\
\text { Manitoba, Saskatchewan e } \\
\text { Alberta, estudo de coorte } \\
\text { retrospectiva. }\end{array}$ & $\begin{array}{l}\text { Analisar a relação entre práticas agrícolas, } \\
\text { variáveis sociodemográficas e o risco } \\
\text { de morte por câncer de próstata entre } \\
\text { agricultores. }\end{array}$ & $\begin{array}{l}\text { A taxa de mortalidade padronizada para câncer de } \\
\text { próstata foi de } 0,92 \text { (IC } 95 \%=0,87-0,98) \text {. Houve } \\
\text { associação fraca, mas estatisticamente significativa } \\
\text { entre o número de acres pulverizados com herbicidas } \\
\text { em } 1970 \text { e o risco de morte por câncer de próstata } \\
(\mathrm{RR}=2,23 ; \text { IC } 95 \%=1,30-3,84 ; \mathrm{p}<0,01) \text {. }\end{array}$ & $\begin{array}{l}\text { Ausência de avaliação da exposição à } \\
\text { outra classe de agrotóxicos. }\end{array}$ \\
\hline
\end{tabular}




\section{Continuação}

\begin{tabular}{|c|c|c|c|}
\hline $\begin{array}{l}\text { Dich; Wiklund, 1998,(31) Suécia, } \\
\text { estudo de coorte prospectiva. }\end{array}$ & $\begin{array}{l}\text { Analisar o risco para câncer de próstata } \\
\text { em indivíduos aplicadores de agrotóxicos } \\
\text { licenciados entre os anos de } 1965 \text { e } 1976 \\
\text { na Suécia. }\end{array}$ & $\begin{array}{l}\text { Houve aumento estatisticamente significativo do risco } \\
\text { de câncer de próstata com uma taxa de incidência } \\
\text { padronizada de } 1,13 \text { (IC } 95 \%=1,02-1,24) \text {. }\end{array}$ & $\begin{array}{l}\text { Ausência de avaliação de fatores de } \\
\text { confusão. }\end{array}$ \\
\hline $\begin{array}{l}\text { Mills; Yang, 2003, }{ }^{(20)} \text { EUA: } \\
\text { Califórnia, estudo de } \\
\text { caso-controle. }\end{array}$ & $\begin{array}{l}\text { Examinar a relação entre a ocorrência } \\
\text { de câncer de próstata e variáveis } \\
\text { ocupacionais. }\end{array}$ & $\begin{array}{l}\text { Houve risco aumentado de câncer de próstata para } \\
\text { agricultores que utilizavam simazina ( } \mathrm{c} O \mathrm{OR}=1,53 ; \\
\text { IC } 95 \%=1,02-2,28) \text {, lindano }(\mathrm{OR}=1,32 ; \mathrm{IC} 95 \%= \\
0,88-1,96) \text {, heptaclor (OR }=1,35 ; \mathrm{IC} 95 \%=0,91-2,01) \text {, } \\
\text { diclorvos (OR }=1,35 ; \mathrm{IC} 95 \%=0,93-1,96) \text { e brometo } \\
\text { de metila (OR }=1,11 ; \mathrm{IC} 95 \%=0,77-1,75) \text {, todos } \\
\text { em altos níveis. Já o aumento do tempo de filiação } \\
\text { sindical diminuiu o risco da ocorrência da patologia } \\
(\mathrm{OR}=0,29 ; \text { IC } 95 \%=0,19-0,45 \text {, para mais de } 95 \\
\text { meses de associação). }\end{array}$ & $\begin{array}{l}\text { Ausência de avaliação do tempo sem } \\
\text { filiação sindical. Provável perda de } \\
\text { trabalhadores migrantes após } \\
\text { diagnóstico. }\end{array}$ \\
\hline $\begin{array}{l}\text { Band et al., 2011, }{ }^{(30)} \text { Canadá: } \\
\text { Colúmbia Britânica, estudo } \\
\text { de caso-controle. }\end{array}$ & $\begin{array}{l}\text { Avaliar o risco de desenvolver câncer } \\
\text { de próstata relacionado à exposição a } \\
\text { agrotóxicos. }\end{array}$ & $\begin{array}{l}\text { Houve associação significativa entre o risco de câncer } \\
\text { de próstata e a exposição ao DDT }(\mathrm{OR}=1,68 ; \mathrm{IC} 95 \% \\
=1,04-2,70) \text {, simazina }(\mathrm{OR}=1,89 ; \mathrm{IC} 95 \%=1,08-3,33) \text {, } \\
\text { lindano }(\mathrm{OR}=2,02 ; \mathrm{IC} 95 \%=1,15-3,55) ; \text { malationa } \\
(\mathrm{OR}=1,34 ; \mathrm{IC} 95 \%=1,01-1,78) ; 2,4 \mathrm{D}(\mathrm{OR}=2,72 ; \\
\mathrm{IC} 95 \%=1,12-6,57) ; \text { captan }(\mathrm{OR}=1,56 ; \mathrm{IC} 95 \%= \\
1,12-2,17) ; \text { carbaril }(\mathrm{OR}=1,54 ; \mathrm{IC} 95 \%=1,08-2,18) ; \\
\text { endossulfan }(\mathrm{OR}=1,52 ; \mathrm{IC} 95 \%=1,00-2,29) ; \text { diazinon } \\
(\mathrm{OR}=1,93 ; \mathrm{IC} 95 \%=1,21-3,08) ; \text { e dicamba }(\mathrm{OR}= \\
2,70 ; \mathrm{IC} 95 \%=1,01 \text { a } 7,20, \text { com base em oito casos } \\
\text { expostos }) .\end{array}$ & $\begin{array}{l}\text { Ausência de informações sobre a } \\
\text { história familiar. Viés de seleção na } \\
\text { classificação da exposição a alguns } \\
\text { agrotóxicos. Viés de recall para } \\
\text { exposição a agrotóxicos específicos. }\end{array}$ \\
\hline $\begin{array}{l}\text { Settimi et al., 2003, }{ }^{(32)} \text { Itália: Asti, } \\
\text { Pescia, Pistoia, Grosseto e Ímola, } \\
\text { estudo de caso-controle. }\end{array}$ & $\begin{array}{l}\text { Avaliar a associação entre o } \\
\text { desenvolvimento de câncer de próstata e } \\
\text { a exposição a agrotóxicos em ambientes } \\
\text { agrícolas na Itália. }\end{array}$ & $\begin{array}{l}\text { Sempre ter trabalhado na agricultura se associou a um } \\
\text { risco aumentado de câncer de próstata }(\mathrm{OR}=1,4 ; \mathrm{IC} \\
95 \%=0,9-2,0) \text {, bem como o uso de agrotóxicos } \\
\text { organofosforados }(\mathrm{OR}=2,5 ; \mathrm{IC} 95 \%=1,4-4,2) \text {. }\end{array}$ & $\begin{array}{l}\text { Número de casos relativamente } \\
\text { reduzido. }\end{array}$ \\
\hline $\begin{array}{l}\text { Meyer } \text { et al., } 2007,{ }^{(23)} \text { EUA: } \\
\text { Carolina do Norte, estudo } \\
\text { de caso-controle. }\end{array}$ & $\begin{array}{l}\text { Determinar se o risco de ocorrência } \\
\text { de câncer de próstata está associado a } \\
\text { atividades agrícolas entre indivíduos } \\
\text { afro-americanos e caucasianos. }\end{array}$ & $\begin{array}{l}\text { A agricultura foi associada ao aumento do risco de } \\
\text { câncer de próstata em caucasianos }(\mathrm{OR}=1,8 ; \mathrm{IC} 95 \% \\
=1,3 \text { a } 2,7) \text {, mas não em afro-americanos }(\mathrm{OR}=1,0 ; \\
\text { IC } 95 \%=0,6 \text { a } 1,6) .\end{array}$ & $\begin{array}{l}\text { Viés de seleção na classificação da } \\
\text { exposição a alguns agrotóxicos. Viés } \\
\text { de recall para exposição a agrotóxicos } \\
\text { específicos. Viés de seleção para } \\
\text { localização de afro-americanos. }\end{array}$ \\
\hline
\end{tabular}




\section{Continuação}

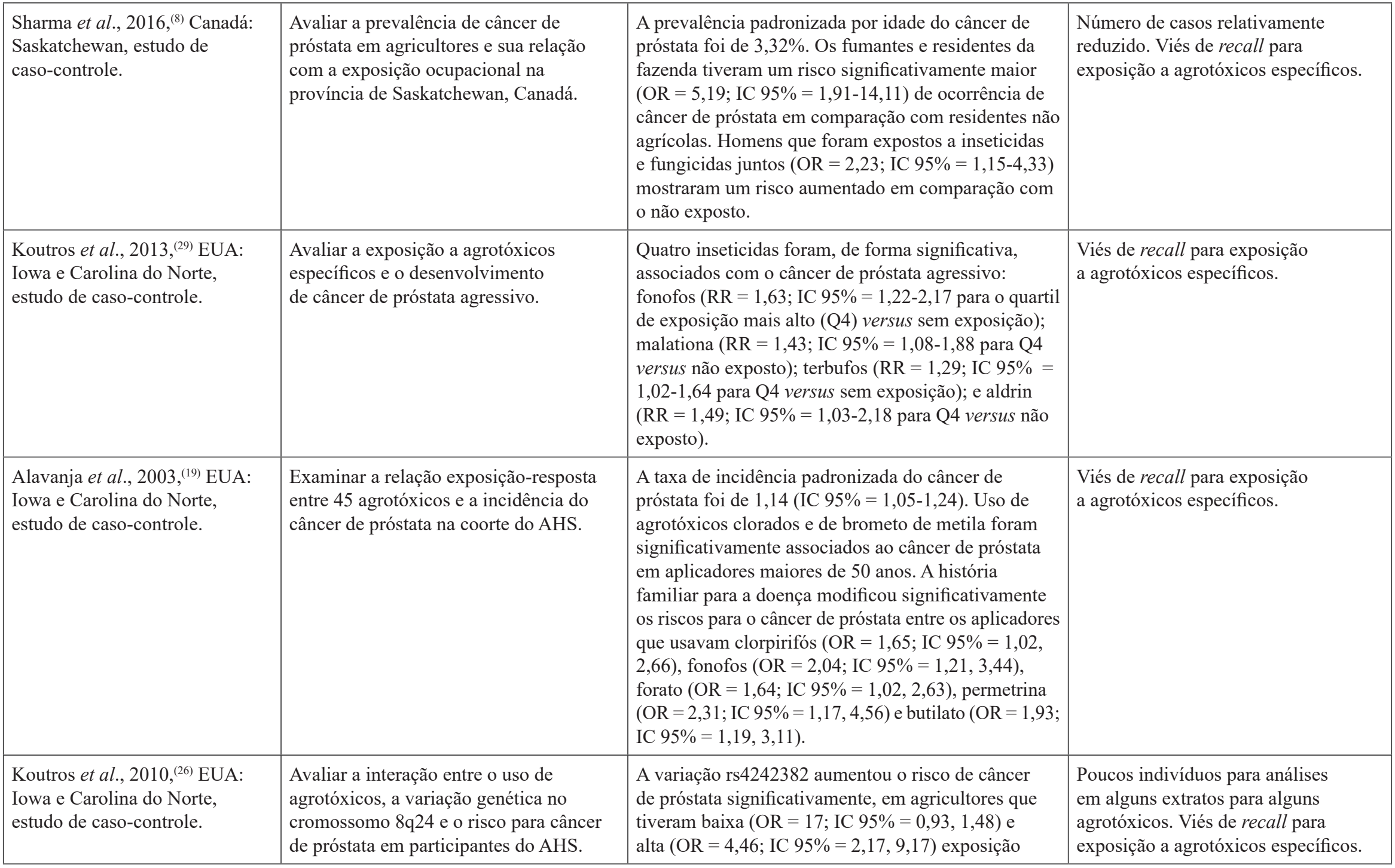




\section{Continuação}

\begin{tabular}{|c|c|c|c|}
\hline & & $\begin{array}{l}\text { ao fonofos. Modificação de efeito semelhante foi } \\
\text { observada para alta exposição a coumafos }(\mathrm{OR}=3,02 \text {; } \\
\text { IC } 95 \%=1,48,6,16) \text {, terbufos }(\mathrm{OR}=2,15 ; \mathrm{IC} 95 \%= \\
1,32-3,52) \text {, permetrina }(\mathrm{OR}=2,73 ; \mathrm{IC} 95 \%=1,31,5,69) \\
\text { e em menor grau para forato (p-interação }=0,26) .\end{array}$ & \\
\hline $\begin{array}{l}\text { Karami et al., 2013, }{ }^{(28)} \text { EUA: } \\
\text { Iowa e Carolina do Norte, } \\
\text { estudo de caso-controle. }\end{array}$ & $\begin{array}{l}\text { Avaliar as interações entre } 41 \text { agrotóxicos } \\
\text { e } 152 \text { polimorfismos de nucleotídeos } \\
\text { únicos (SNPs) em nove genes da via da } \\
\text { vitamina D em relação à ocorrência de } \\
\text { câncer de próstata. }\end{array}$ & $\begin{array}{l}\text { Foram observadas interações entre o terbufos }(\mathrm{OR}= \\
1,73 ; \mathrm{IC} 95 \%=1,20-2,49 \text { alta versus sem exposição) } \\
\text { e o paration }(\mathrm{OR}=3,09 ; \mathrm{IC} 95 \%=1,10-8,68 \text {, alta } \\
\text { versus sem exposição) e variações genéticas nos } \\
\text { genes da via da vitamina D (VDR, RXRB e CG), } \\
\text { resultando em aumento do risco de ocorrência do } \\
\text { câncer de próstata. }\end{array}$ & $\begin{array}{l}\text { Casos reduzidos devido à baixa } \\
\text { qualidade da amostra de saliva. } \\
\text { Viés de recall para exposição } \\
\text { a agrotóxicos específicos. }\end{array}$ \\
\hline $\begin{array}{l}\text { Barry et al., 2012,(5) EUA: Iowa } \\
\text { e Carolina do Norte, estudo de } \\
\text { caso-controle. }\end{array}$ & $\begin{array}{l}\text { Avaliar as interações entre a exposição } \\
\text { a agrotóxicos e a variação genética em } \\
27 \text { genes da via de reparo por excisão } \\
\text { de nucleotídeos (NER) em relação à } \\
\text { ocorrência de câncer de próstata. }\end{array}$ & $\begin{array}{l}\text { Homens com o alelo variante A no ERCC1 rs } 2298881 \\
\text { exibiram aumento no risco de câncer de próstata com } \\
\text { alto versus nenhum uso de fonofos (OR = 2,98; IC } \\
95 \%=1,65-5,39) \text {. Homens carregando o genótipo } \\
\text { homozigoto TT do tipo selvagem em dois SNPs } \\
\text { (rs11744596 e rs } 2932778 \text { ) CDK } 7 \text { correlacionados com } \\
\text { alto versus nenhum uso de carbofurano apresentaram } \\
\text { risco aumentado para câncer de próstata (OR = 2,01; } \\
\text { IC } 95 \%=1,31-3,10) \text {. }\end{array}$ & $\begin{array}{l}\text { Casos reduzidos devido à baixa } \\
\text { qualidade da amostra de saliva. } \\
\text { Número reduzido de participantes } \\
\text { quando estratificado por variedade } \\
\text { homozigotas. Viés de recall para } \\
\text { exposição a agrotóxicos específicos. }\end{array}$ \\
\hline $\begin{array}{l}\text { Barry et al., 2011, }{ }^{(27)} \text { EUA: Iowa } \\
\text { e Carolina do Norte, estudo de } \\
\text { caso-controle. }\end{array}$ & $\begin{array}{l}\text { Avaliar as interações entre exposições a } \\
\text { agrotóxicos e a variação genética em } 31 \\
\text { genes da via de reparo por excreção de } \\
\text { bases (BER) em relação à ocorrência } \\
\text { de câncer de próstata. }\end{array}$ & $\begin{array}{l}\text { A exposição a fonofos foi associada a um aumento no } \\
\text { risco de ocorrência de câncer de próstata entre homens } \\
\text { com os genótipos CT } / \text { TT do SNP rs } 1983132 \text { de NEIL3 } \\
\text { para baixo }(\mathrm{OR}=1,65 ; \text { IC } 05 \%=0,91-3,01) \text { e alto uso } \\
(\mathrm{OR}=3,25 ; \text { IC } 05 \%=1,78-5,92) \text { quando comparados } \\
\text { com indivíduos que não usavam fonofos. }\end{array}$ & $\begin{array}{l}\text { Casos reduzidos devido à baixa } \\
\text { qualidade da amostra de saliva. } \\
\text { Número reduzido de participantes } \\
\text { quando estratificado por variedade } \\
\text { homozigotas. Viés de recall para } \\
\text { exposição a agrotóxicos específicos. }\end{array}$ \\
\hline $\begin{array}{l}\text { Silva et al., 2015, }{ }^{(9)} \text { Brasil, estudo } \\
\text { ecológico. }\end{array}$ & $\begin{array}{l}\text { Explorar correlações entre variáveis } \\
\text { relativas à produção agrícola, ao uso de } \\
\text { serviços de saúde e sociodemográficas } \\
\text { e às taxas de mortalidade por câncer de } \\
\text { próstata entre } 2005 \text { e } 2009 \text { nos estados } \\
\text { brasileiros. }\end{array}$ & $\begin{array}{l}\text { As variáveis que apresentaram associação com a taxa } \\
\text { de mortalidade por câncer de próstata foram: toneladas } \\
\text { de soja produzidas }(p=0,030) \text {; proporção da população } \\
\text { com } 80 \text { anos e mais }(p<0,001) \text {; e consumo de bebidas } \\
(p<0,001) \text {. }\end{array}$ & $\begin{array}{l}\text { Viés de agregação. Falácia ecológica. } \\
\text { Heterogeneidade de qualidade dos } \\
\text { dados referentes ao Sistema de } \\
\text { Informação de Mortalidade (SIM). }\end{array}$ \\
\hline
\end{tabular}

Legenda: ${ }^{\mathrm{R} R}$ : risco relativo; ${ }^{\mathrm{H} I C}$ : intervalo de confiança; ${ }^{\mathrm{c}} \mathrm{OR}$ : razão de chances/odds ratio.

Fonte: os pesquisadores, 2020. 
Foram identificadas associações entre a exposição de trabalhadores rurais a agrotóxicos e a ocorrência de câncer de próstata em 17 artigos $(89,47 \%) .{ }^{(5,8-9,12,18-20,23-32)}$ Dois trabalhos $(10,52 \%)$ realizaram a avaliação de agrotóxicos específicos, fonofos ${ }^{(22)}$ e cianazina, ${ }^{(21)}$ e não verificaram evidências entre a exposição a estes produtos e a ocorrência de câncer de próstata. Os resultados variaram consideravelmente de acordo com a região geográfica e o desenho do estudo.

\section{Discussões}

Foram identificadas associações entre atividades na agricultura e a ocorrência de câncer de próstata em 17 artigos $(89,47 \%) .^{(5,8-9,12,18-20,23-32)}$ Nestes, foram identificados os seguintes fatores de risco relacionados à exposição a agrotóxico: histórico familiar, ${ }^{(18-19,22,24-25)}$ raça, ${ }^{(23)}$ idade, ${ }^{(8-9,18-19,23,32)}$ tabagismo, ${ }^{(8)}$ consumo de bebidas alcoólicas, ${ }^{(9)}$ alterações genéticas, ${ }^{(5,26-28)}$ número de acres pulverizados com agrotóxicos, ${ }^{(17)}$ produções de milho e soja ${ }^{(9)}$ e o uso de agrotóxicos específicos. ${ }^{(5,19-22,24-30,32)}$

O histórico familiar de câncer é um fator de risco mundialmente reconhecido para uma série de localizações primárias de cânceres, inclusive o de próstata. Estudos, inclusos nesta revisão, mostram o efeito da história familiar sobre o risco relativo e a razão de chances de ocorrência de câncer de próstata. ${ }^{(18-19,22,24-25)}$ Corroborando estes resultados, Silva, ${ }^{(4)}$ em tese de doutorado, verificou aumento na razão de chance de mortalidade por câncer de próstata em homens com história familiar deste agravo $(\mathrm{OR}=2,24$; IC 95\% = 1,39-3,61). Tal especificidade sugere a possibilidade de genes familiares aumentarem a suscetibilidade a fatores de risco entre os membros da família, servindo de indicativo para relação da patologia com características genéticas herdadas. O acréscimo significativo da ocorrência deste câncer em expostos a determinadas classes químicas de agrotóxicos, como, por exemplo, os organofosforados, dá mais apoio à hipótese. $^{(19)}$

Já a raça pode estar concatenada à predisposição genética e ao estilo de vida. ${ }^{(33)}$ Somente o estudo de Meyer et al. ${ }^{(23)}$ avaliou especificamente a raça à exposição a agrotóxicos, encontrando risco aumentado para câncer de próstata entre brancos $(\mathrm{OR}=1,8 ; \mathrm{IC}=95 \% 1,3$ a 2,7) e ausência em afrodescendentes (OR $=1,0$; IC 95\% $=0,6$ a 1,6). Embora os afrodescendentes tenham altas taxas de incidência de câncer de próstata, ${ }^{(34)}$ a maioria dos estudos avaliou a associação desta patologia com a agricultura apenas em homens de cor branca. Ressalta-se a dificuldade de avaliação da variável raça/cor, devido ao número limitado de agricultores negros em algumas regiões dos estudos.

O envelhecimento é considerado um importante fator de risco para o desenvolvimento do câncer de próstata. ${ }^{(35)}$ Nesta revisão, seis estu$\operatorname{dos}^{(8-9,18-19,23,32)}$ relacionaram a idade aumentada ao elevado risco para câncer de próstata em agricultores expostos a agrotóxicos. Destarte, ratificando esses resultados, outros estudos encontraram maior risco relativo de câncer de próstata entre agricultores mais velhos. ${ }^{(36-37)}$ Contudo, há um reduzido número de agricultores jovens que sofrem de câncer de próstata, o que pode levar a estimativas imprecisas. Portanto, sugere-se que pode haver a coexistência de outros fatores, além do envelhecimento, interferindo no padrão verificado.

$\mathrm{O}$ tabagismo foi indicado como fator de risco no estudo de Sharma et al..$^{(8)}$ Kenfield et al.$^{(7)}$ também associaram o consumo de maior número de cigarro por ano ao risco aumentado de mortalidade por câncer de próstata $(\mathrm{OR}=1,61$; IC 95\% = 1,11-2,32). Entre as hipóteses biologicamente plausíveis para esta relação há a promoção de tumores por cancerígenos da fumaça do tabaco, como o cádmio. ${ }^{(38)}$ Anetor et al. ${ }^{(39)}$ encontraram em tabagistas um nível sérico de cádmio três vezes maior que em não fumantes. Esta substância pode interferir no processo de carcinogênese, através de modificações no DNA celular e indução de estrese oxidativo. ${ }^{(38)}$ Por outro lado, estudo realizado na Austrália não encontrou esta associação em tabagistas atuais, indivíduos com história pregressa de tabagismo e os que pararam de fumar entre 11 e 20 anos antes do desenvolvimento do câncer de próstata, ${ }^{(40)} \mathrm{o}$ que indica a necessidade de mais pesquisas sobre o tema.

Silva et al. ${ }^{(9)}$ indicaram o consumo de bebidas como um fator de risco para o desenvolvimento 
de câncer de próstata em agricultores expostos a agrotóxicos no Brasil. Gong et al. ${ }^{(41)}$ verificaram que o elevado consumo de álcool diário (50 g de álcool por dia) e o consumo regular de bebidas alcoólicas ( 4 bebidas por dia em 5 dias por semana) estavam associados a riscos aumentados de câncer de próstata $(\mathrm{RR}=2,01$; IC 95\% = 1,33-3,05). Porém, apesar do número de pesquisas que buscam indicar associações entre hábitos alimentares e o câncer de próstata, esta relação ainda é inconclusiva.

$\mathrm{O}$ uso de agrotóxicos específicos também foi vinculado ao desenvolvimento do câncer de próstata. Entre os artigos inclusos nesta revisão, associações foram observadas para os inseticidas organofosforados fosfonos, ${ }^{(5,19,26-27,29)}$ coumafós, ${ }^{(25-26)}$ malationa, ${ }^{(29-30)}$ terbufós, ${ }^{(26,28-29)}$ forato, ${ }^{(19,26)}$ paration, ${ }^{(28)}$ diazinon $^{(30)}$ e clorpirifós; ${ }^{(19)}$ para os inseticidas organoclorados lindano, ${ }^{(20,30)}$ heptaclor, ${ }^{(20)}$ $\mathrm{DDT}^{(30)}$ e aldrin; ${ }^{(29)}$ para os inseticidas carbamatos carbofurano $^{(5)}$ e carbaril, ${ }^{(30)}$ e o inseticida alifático halogenado brometo de metila; ${ }^{(19)}$ para o inseticida piretróide permetrina; ${ }^{(19)}$ inseticida ciclodienoclorado endosulfan; ${ }^{(30)}$ para o herbicida tiocarbamato butilato, ${ }^{(19,24)}$ herbicidas da classe química triazina, simazina ${ }^{(30)}$ e cianazina, ${ }^{(20)}$ além do fungicida dicarboximida captan. ${ }^{(30)}$

Estudos indicam que há agrotóxicos, particularmente os organofosforados e organoclorados, que possuem potencial genotóxico e de disrupção endócrina, ${ }^{(6,12)}$ indicando a plausibilidade biológica da exposição a estes produtos frente à ocorrência do câncer de próstata. Substâncias genotóxicas interagem com o DNA e podem causar uma série de danos, havendo a possibilidade de iniciar as primeiras etapas da carcinogênese da próstata. ${ }^{(12,42)} \mathrm{Nesta}$ revisão, Barry et al. ${ }^{(27)}$ e Barry et al. ${ }^{(5)}$ encontraram elevada razão de chance de desenvolvimento de câncer de próstata relacionada a variações genéticas de genes envolvidos na via de reparo por excisão de nucleotídeos (NER) e na via de reparo por excreção de bases (BER), nesta ordem. A plausibilidade, nestes casos, deduz-se do fato de que estas vias são importantes na reparação de danos oxidativos ao DNA. ${ }^{(43)}$ Outros estudos de biomonitoramento humano observaram um aumento de danos genéticos (troca de cromátides irmãs, formação de micronúcleos) causados pela exposição a misturas de agrotóxicos. ${ }^{(43-44)}$

Já Karami et al. ${ }^{(28)}$ encontraram risco aumentado para câncer de próstata entre os agricultores com variações genéticas nos genes da via da vitamina $\mathrm{D}$ expostos a terbufós e paration. Infere-se que essa vitamina e seus metabólitos possam impedir a carcinogênese através da estimulação da diferenciação celular, inibindo a proliferação celular, induzindo apoptose, suprimindo a invasão tumoral, angiogênese e metástase, bem como reduzindo o estresse oxidativo e a inflamação. ${ }^{(45)}$ Indica-se, portanto, que danos genéticos que envolvam vitamina D poderiam prejudicar este processo de proteção.

Ainda, alterações hormonais são postuladas como mecanismos envolvidos no processo de desenvolvimento do câncer de próstata. ${ }^{(46)}$ Há evidências de que contaminantes químicos, especialmente os agrotóxicos organofosforados e organoclorados, são disruptores endócrinos capazes de aumentar a produção de hormônios androgênicos ou imitar sua ação. ${ }^{(47)}$ Andrógenos, particularmente a testosterona e a di-hidrotestosterona, são importantes para crescimento e funcionamento normais da próstata; variações nestes níveis hormonais podem gerar efeitos adversos como hiperplasia ou o câncer. ${ }^{(48-49)}$ Entretanto, ainda não está claro como esses mecanismos ocorrem na próstata humana e como eles desencadeiam câncer de próstata.

Kumar et al. ${ }^{(12)}$ verificaram níveis elevados de agrotóxicos organoclorados em pacientes portadores de câncer de próstata, inferindo o possível papel desses compostos como uma das causas desta doença. Já Lynch et al. ${ }^{(27)}$ não verificaram risco relativamente maior para o câncer de próstata $(\mathrm{RR}=$ 1,23 ; IC 95\% = 0,87-1,70) em agricultores expostos à cianazina. Mahajan et al. ${ }^{(22)}$ não constataram aumento deste risco em agricultores que tinham histórico familiar para câncer de próstata e que foram expostos a fonofos. Apesar da falta de homogeneidade da literatura sobre o tema, ressalta-se que, em geral, as atividades agrícolas são exercidas em um ambiente de exposição a agrotóxicos com acumulação a longo prazo, os quais podem acarretar maiores riscos de interferências na função hormonal normal e de danos ao DNA. ${ }^{(43-44)}$ 
Avaliações de múltiplas exposições devem ser consideradas em um cenário realista. Trabalhadores rurais podem se expor a várias formulações e princípios ativos conjuntamente ao longo dos anos, sob grande variedade de condições. ${ }^{(14)}$ São expostos através do transporte, carregamento, preparo, aplicação e limpeza de equipamentos, com frequências intermitentes, ${ }^{(14)}$ levando a quantificações de exposições diversificadas em diferentes realidades. Ademais, há agrotóxicos que resistem à degradação, bioacumulando em tecidos adiposos de humanos e persistindo no meio ambiente.

Semitti et al. ${ }^{(32)}$ encontraram risco elevado de câncer de próstata entre homens que relataram utilizar misturas de agrotóxicos (OR = 1,7; IC 95\% $=1,2-2,6)$. Sharma et al. ${ }^{(8)}$ avaliaram simultaneamente mais de uma substância, identificando risco de aumento para câncer de próstata entre os expostos a inseticidas e fungicidas juntos $(\mathrm{OR}=2,23$; IC $95 \%=1,15-4,33, \mathrm{p}=0,02)$. O poder limitado das demais pesquisas inclusas nesta revisão ${ }^{(20-22,25-26,28-30)}$ impediu ou prejudicou a investigação do risco relacionado às combinações de agrotóxicos.

As toneladas de soja e milho produzidas e o número de acres pulverizados com agrotóxicos também foram relacionados à mortalidade por câncer de próstata nos estudos de Silva et al ${ }^{(9)} \mathrm{e}$ Morrison et al. ${ }^{(17)}$ Os cultivos de soja e milho são marcados pelo uso extenso de terras, mecanização do plantio e da colheita e pelo uso elevado de agrotóxicos. Estas características contribuem para a exposição de trabalhadores rurais, moradores dos arredores de áreas agricultáveis e da população geral, que pode consumir água e alimentos contaminados, a agrotóxicos. ${ }^{(49)}$ No município de Lucas do Rio Verde, estado de Mato Grosso, Brasil, foi detectado que $88 \%$ das amostras de sangue coletadas de trabalhadores e residentes locais estavam contaminadas com glifosato; além disso, $61 \%$ das amostras de urina apresentaram resíduos de pelo menos um tipo de inseticida organoclorado. ${ }^{(50)} \mathrm{Em}$ sua tese, Silva ${ }^{(4)}$ identificou associação entre as toneladas de soja produzidas com a elevação da taxa de mortalidade por câncer de próstata $(\mathrm{p}=0,030)$ em residentes do estado de Mato Grosso do Sul, Brasil.
Destaca-se que as práticas agrícolas e o uso de agrotóxicos podem diferir entre regiões e países, de modo que avaliações sobre suas consequências para a saúde humana devem ser realizadas localmente. Em Mato Grosso, por exemplo, entre os 50 agrotóxicos mais consumidos, há 20 agrotóxicos para os quais existem evidências epidemiológicas de que são desreguladores endócrinos, mutagênicos e teratogênicos. ${ }^{(50)}$

Os artigos avaliados apresentam algumas limitações; é possível existir viés de seleção para determinar a exposição a agrotóxicos específi$\cos ,{ }^{(23,30)}$ uma vez que os trabalhadores rurais raramente são expostos a somente uma substância, tornando imprecisa esta determinação. Isso pode ser intensificado pelo viés de recall, decorrente da perda de informações difíceis de serem recordadas devido à passagem do tempo, além do conhecimento incompleto sobre todas as exposições. ${ }^{(5,8,19,21-28,30)}$ Ressalta-se que há esforços em andamento para o monitoramento das exposições na coorte do AHS, o que poderá refinar as estimativas futuras.

O emprego do estudo ecológico ${ }^{(9)}$ tem seu viés em relação ao fato de que a observação da existência de uma relação entre duas variáveis no nível agregado não implica, necessariamente, que essa relação se mantenha no nível individual. O controle de fatores de confusão também foi um problema nas análises, , $^{(9,17-18,20,30-32)}$ tendo em vista que idade, origem étnica, histórico familiar e estilo de vida (hábitos alimentares, tabagismo, etilismo, entre outros) podem modificar o efeito de determinantes ambientais e ocupacionais. Além disso, nos estudos sobre fatores genéticos, ${ }^{(5,27-28)}$ a coleta de amostra biológica pelos próprios agricultores pode ter contribuído para um número relativamente elevado de amostras com má qualidade.

Como pontos fortes, pode-se dizer que os resultados apresentam plausibilidade para a ocorrência de câncer de próstata em indivíduos expostos a agrotóxicos que exercem atividades agrícolas. Ademais, nossos dados fornecem uma visão considerável sobre fatores de risco para câncer de próstata em trabalhadores rurais expostos a agrotóxicos. A partir das variáveis analisadas foram identificadas carências de artigos realizados em regiões com 
médio e baixo índice de desenvolvimento humano, de pesquisas nacionais, com metodologias que permitam a análise de dados individuais, mensurações da exposição confiável, que incluam investigações minuciosas dos compostos químicos envolvidos neste processo e que avaliem a intensidade da exposição. Como limitações deste trabalho, inclui-se a prevalência de países com índice de desenvolvimento humano muito alto, o que não permitiu a análise de resultados entre sistemas agrícolas de nações com alto, médio e baixo desenvolvimento humano. Houve também reduzido número de pesquisas nacionais.

\section{Conclusão}

Nesta revisão, foram analisados 19 artigos, verificando-se associações entre o trabalho agrícola, a exposição a agrotóxicos e a ocorrência de câncer de próstata em 17 deles. Identificou-se como fatores de risco o histórico familiar, o tabagismo, a raça, a idade avançada, o consumo de bebidas alcoólicas, as alterações genéticas, o número de acres pulverizados com agrotóxicos, as produções de milho e soja e o uso de agrotóxicos específicos.

Dois artigos não encontraram evidências da relação abordada. Contudo, salienta-se que estes realizaram avaliações sobre exposições a substâncias específicas, o que pode não corresponder à realidade vivenciada pelos trabalhadores do setor agropecuário, os quais são expostos a múltiplas substâncias durante o processo laboral.

Nossos resultados inferem um papel biológico plausível para a ocorrência de câncer de próstata em trabalhadores rurais expostos a agrotóxicos, reforçando a sugestão de que esta exposição pode ser um fator causal comum nesta população, para a patologia estudada. Espera-se, a partir dos resultados desta pesquisa, motivar políticas de vigilância às populações expostas a agrotóxicos a fim de proporcionar a prevenção e promoção da saúde deste público.

Para estudos futuros, sugere-se que métodos de pesquisa devem ser expandidos a fim de se empregar estratégias que possibilitem a coleta e análise de dados individuais, mensuração fidedigna das exposições, sua intensidade e os compostos químicos envolvidos no processo. Pesquisas sobre o tema em países com médio ou baixo índice de desenvolvimento humano e nacionais precisam ser incentivadas.

\section{Referências}

1 Bray F, Ferlay J, Soerjomataram I, Siegel RL, Torre LA, Jemal A. Global cancer statistics 2018: GLOBOCAN estimates of incidence and mortality world wide for 36 cancers in 185 countries. CA Cancer J Clin. 2018;68(6):394424. doi: 10.3322/caac. 21492.

2 Instituto Nacional de Câncer José Alencar Gomes da Silva (INCA). Estimativa 2020: incidência de câncer no Brasil. Rio de Janeiro; 2019.

3 Stewart BW, Wild CP. World cancer report 2014. Lyon: IARC Press; 2014.

4 Silva JFS. Câncer de Próstata e Exposição a Agrotóxicos no Estado de Mato Grosso do Sul. [tese]. Campo Grande: Universidade Federal de Mato Grosso do Sul; 2015.

5 Barry KH, Koutros S, Andreotti G, Sandler DP, Burdette AL, Yeager M, et al. Genetic variation in nucleotide excision repair pathway genes, pesticide exposure and prostate cancer risk. Carcinogenesis. 2012; 33(2):331-37. doi: 10.1093/carcin/bgr258.

6 Badajoz ES, Lage-Sánchez JM, SánchezGallegos P. Disruptores endócrinos e câncer de próstata. Arquivo Espanhol de Urologia. [Internet] 2017. [citado $2020 \mathrm{Abr} 03$ ]; 70(3):331-635. Disponible en: https://ialnet.unirioja.es/servlet/ articulo? codigo $=5924963$

7 Kenfield SA, Stampfer MJ, June M, Chan JM, Giovannucci E. Smoking and Prostate Cancer Survival and Recurrence. JAMA. [Internet] 2011. [cited 2020 Mar 9]; 305(24):2548. Available from: https://jamanetwork.com/journals/jama/ fullarticle/646754

8 Sharma M, Lawson JA, Kanthan R, Karunanayake C, Hagel L, Rennie D, et al. Factors Associated With the Prevalence of Prostate Cancer in Rural Saskatchewan: The Saskatchewan Rural Health Study. The Journal of Rural Health. 
[Internet] 2016. [cited 2020 Feb. 12]; 32:12535. Available from: https://onlinelibrary.wiley. com/doi/abs/10.1111/jrh.12137

9 Silva JFA, Silva MAS, Lima-Luz L, Aydos RD, Mattos IE. Correlação entre produção agrícola, variáveis clínicas-demográficas e câncer de próstata: um estudo ecológico. Ciência \& Saúde Coletiva. [Internet] 2015. [citado 2020 fev. 12]; 20(9):2805-12. Disponível em: https://www.scielo.br/scielo.php?pid=S1413$81232015000902805 \& \mathrm{script}=\mathrm{sci}$ arttext\&tlng=pt

10 Ministério da Saúde - MS (BR). Secretaria de Atenção à Saúde. Departamento de Ações Programáticas Estratégicas. Protocolo de Atenção à Saúde dos Trabalhadores Expostos a agrotóxicos. Brasília: Ministério da Saúde; 2016.

11 Preza DLC, Augusto LGS. Vulnerabilidades de trabalhadores rurais frente ao uso de agrotóxicos na produção de hortaliças em região do Nordeste do Brasil. Revista Brasileira de Saúde Ocupacional. [Internet] 2012. [citado 2020 maio 04]; 37(125): 89-98. Disponível em: https://www.scielo.br/scielo.php?pid=S0303$76572012000100012 \& \mathrm{script}=\mathrm{sci}$ abstract\&tlng $=\mathrm{pt}$

12 Kumar V, Yadav CS, Singh S, Goel S, Ahmed RS, Gupta S, Grover RK, et al. CY1P1 polymorphism and organoclorine pesticides leves in the etiology of prostate cancer. Chemosphere. [Internet] 2010. [cited 2020 Mar. 12]; (81):464-68. Available from: https://www. sciencedirect.com/science/article/abs/pii/ S0045653510008805?via\%3Dihub

13 Pignati WA, Oliveira NP, Silva AMC. Vigilância aos agrotóxicos: quantificação do uso e previsão de impactos na saúde trabalho ambiente para os municípios brasileiros. Revista Ciência e Saúde Coletiva. [Internet] $2014 \mathrm{dez}$. [citado 2020 fev 20]; 19(12):4669-78. Disponível em: https://www.scielo.br/scielo.php?script=sci abstract\&pid $=$ S1413-81232014001204669\&ln $\mathrm{g}=\mathrm{pt} \& \mathrm{nrm}=$ iso $\&$ tlng $=\mathrm{pt}$

14 Ruths JC, Rizzoto MLF, Machineski GG. Exposure to pesticides and cancer occurrence in workers of two municipalities of West Paraná. Ciência, Cuidado e Saúde. 2019; 18(3). doi: 10.4025/cienccuidsaude.v18i3.44570.
15 Joanna Briggs Institute (JBI). Methodology for JBI Scoping Reviews - Joanna Briggs 2015. [Internet] Australia: JBI; 2015. [cited 02 Jan. 2020]. Available from: http://joannabriggs. org/assets/docs/sumari/Reviewers Manual_Methodology-for-JBI-ScopingReviews_2015_v2.pdf

16 Marziale MHP. Instrumento para recolección de datos revisión integrativa. [Internet] 2017. [citado 18 jan. 2020]. Disponible en: http:// gruposdepesquisa.eerp.usp.br/sites/redenso/ publicaciones/

17 Morrison H, Savitz D, Semenciw R, Hulka B, Mao Y, Morindon D, et al. Farming e prostate cancer mortality. Am J Epidemiol. 1993; 137(3):270-80. doi: 10.1093/oxfordjournals. aje.a116674.

18 Parker AS, James RC, Putnam SD, Cantor $\mathrm{KP}$, Lynch CF. A cohort study of farming and risk of prostate cancer in Iowa. Epidemiology. 1999; 10(4):453-68. doi: 10.1097/00001648199907000-00016.

19 Alavanja MCR, Samanic C, Dosemeci M, Lubin J, Tarone R, Lynch CF, et al. Use of Agricultural Pesticides and Prostate Cancer Risk in the Agricultural Health Study Cohort. Am J Epidemiol. [Internet] 2003. [cited 2020 Feb 12]; 157:800-14. doi: 10.1093/aje/kwg040.

20 Mills PK, Yang R. Prostate Cancer Risk in California Farm Workers. J Occup Environ Med. [Internet] 2003. [cited 2020 Feb 12]; 45:249-58. Available from: https://europepmc. org/article/med/12661182

21 Lynch SM, Rusiecki JA, Blair A, Dosemeci $\mathrm{M}$, Lubin J, Sandler D, et al. Cancer Incidence among Pesticide Applicators Exposed to Cyanazine in the Agricultural Health Study. Environ Health Perspect. 2006; 114:1248-52. doi: 10.1289/ehp.8997.

22 Mahajan R, Blair A, Lynch CF, Schroeder P, Hoppin JA, Alavanja MCR. Fonofos Exposure and Cancer Incidence in the Agricultural Health Study. Environ Health Perspect. 2006; 114: 1838-42. doi: 10.1289/ehp.9301.

23 Meyer TE, Coker AL, Sanderson M, Symanski E. A case-control study of farming and prostate 
cancer in African-American and Caucasian men. Occup Environ Med. [Internet] 2007. [cited 2020 Feb 12]; 64:155-60. Available from: https://oem.bmj.com/content/64/3/155.long

24 Lynch SM, Mahajan R, Freeman LEB, Hoppin JA, Alavanja MC. Cancer incidence among pesticide applicators exposed to butylate in the agricultural health study (AHS). Environ Res. 2009 Out; 109(7):860-68. doi: 10.1016/j. envres.2009.06.006.

25 Christensen CH, Platz EA, Andreotti G, Blair A, Hoppin JA, Koutros S, et al. Coumaphos Exposure and Incident Cancer among Male Participants in the Agricultural Health Study (AHS). Environ Health Perspect. [Internet] 2010. [cited 2020 feb 12]; 118:92-96. doi: 10. 1289/ehp.0800446.

26 Koutros S, Freeman LEB, Berndt SI, Andreotti G, Lubin JH, Sandler DP, et al. Pesticide use modifies the association between genetic variants on chromosome $8 \mathrm{q} 24$ and prostate cancer. Cance Res. 2010; 70(22):9224-33. doi: 10.1158/0008-5472.CAN-10-1078.

27 Barry KH, Koutros S, Berndt SI, Andreotti G, Hoppin JA, Sandler DP, et al. Genetic Variation in Base Excision Repair Pathway Genes, Pesticide Exposure, and Prostate Cancer Risk. Environ Health Perspect. [Internet] 2011. [cited 2020 Feb 12]; 119:1726-32. Available from: https://ehp.niehs.nih.gov/doi/10.1289/ ehp. 1103454

28 Karami S, Andreotti G, Koutros S, Barry KH, Moore LE, Han S, et al. Pesticide exposure and inherited variants in vitamin D pathway genes in relation to prostate cancer. Cancer Epidemiol Biomarkers Prev. 2013 Sep.; 22(9):1557-66. doi: 10.1158/1055-9965.EPI-12-1454.

29 Koutros S, Freeman LEB, Lubin JH, Heltshe SL, Andreotti G, Barry KH, et al. Risk of Total and Aggressive Prostate Cancer and Pesticide Use in the Agricultural Health Study. Am J Epidemiol. [Internet] 2013. [cited 2020 Feb. 12]; 177(1):59-74. Available from: https:// academic.oup.com/aje/article/177/1/59/129050

30 Band PR, Abanto Z, Bert J, Lang B, Gallagher RP. Prostate Cancer Risk and Exposure to Pesticides in British Columbia Farmers. The
Prostate. [Internet] 2011. [cited 2020 Feb 12]; 71:168-83. Available from: https://onlinelibrary. wiley.com/doi/abs/10.1002/pros.21232

31 Dich J, Wiklund K. Prostate Cancer in Pesticide Applicators in Swedish Agriculture. The Prostate. [Internet] 1998. [cited 2020 Feb 12]; 34:10012. Available from: https://onlinelibrary.wiley. com/doi/abs/10.1002/\%28SICI\%291097$0045 \% 2819980201 \% 2934 \% 3$ A2\%3C100 $\% 3 \mathrm{~A} \% 3$ AAID-PROS4\%3E3.0.CO\%3B2O?sid=nlm\%3Apubmed

32 Settimi L, Masina A, Andrion A, Axelson O. Prostate cancer and exposure to pesticides in agricultural settings. Int $\mathrm{J}$ Cancer. [Internet] 2003. [cited 2020 Feb 12]; 104, 458-61. Available from: https://onlinelibrary.wiley.com/doi/ full/10.1002/ijc. 10955

33 Van Czorny RCN, Pinto MH, Pompeo DA, Barreta D, Cardoso LV, Silva DM. Fatores de risco para o câncer de próstata: população de uma Unidade Básica de Saúde. Cogitare Enferm. [Internet] 2017. [citado 2020 mar 15]; (22)4: e51823. Disponível em: https://revistas. ufpr.br/cogitare/article/view/51823/pdf

34 Azal Neto W. Impacto do comprimento do fragmento da biópsia transretal no subestadiamento do câncer de próstata em pacientes negros. [dissertação]. Campinas (SP): Universidade Estadual de Campinas; 2019.

35 Franceschi S, La Vecchia C. Cancer epidemiology in the elderly. Critical Reviews in Oncology/Hematology. [Internet] 2001. [cited 2020 May 02]; (39):219-26. Available from: https://www.sciencedirect.com/science/article/abs/pii/S1040842801001020?via\%3Dihub

36 Lima LR, Silva ILC, Alves DC. Investigação e prevalência dos fatores de risco para elevação e desenvolvimento de câncer de próstata e elevação do PSA: uma revisão de literatura. Rev Interd Ciênc Saúde. [Internet] 2017. [citado 2020 maio 16]. 4(1):11-16. Disponível em: https://comunicata.ufpi.br/index.php/rics/ article/view/6723/3890

37 Sarris AB, Candido FJLF, Filho CRP, Staichak BP. Câncer de próstata: uma breve revisão atualizada. Visão Acadêmica. [Internet] 2018. [citado 2020 maio 20]; 19(1):137-51. Disponível 
em: https://revistas.ufpr.br/academica/article/ view/57304. Acesso em: 07 jul. 2021.

38 Enokida A, Shiina H, Urakami S, Terashima M, Ogishima T, Long-Cheng, et al. Smoking influences aberrant $\mathrm{CpG}$ hypermethylation of multiple genes in human prostate carcinoma. Cancer. [Internet] 2006. [cited 2020 Mar 19]; 106(1):79-86. Available from: https:// acsjournals.onlinelibrary.wiley.com/doi/ full/10.1002/cncr.21577

39 Anetor JI, Ajose F, Anetor GO, Iyanda AA, Babalola BB, Adeniyi FAA. High cadmium/ zinc ratio in cigarette smokers: potential implications as a biomarker of risk of prostate cancer. Nigerian Journal of Physiological Sciences. 2008; 23(1-2):41-9. doi: 10.4314/ njps.v23i1-2.54921.

40 Fritschi L, Glass DC, Tabrizi JS, Leavy JE, Ambrosini GL. Occupational risk factors for prostate cancer and benign prostatic hyperplasia: a case-control study in Western Australia. Occup Environ Med. 2007; 64:60-65. doi: 10.1136/oem.2006.027706.

41 Gong Z, Kristal AR, Schenk JM, Tangen CM, Goodman PJ, Thompson IM. Alcohol consumption, finasteride, and prostate cancer risk. Cancer. [Internet] 2009. [cited 2020 May 02]; 115(16), 3661-69. Available from: https://www. ncbi.nlm.nih.gov/pmc/articles/PMC2739798/

42 Klauning JE, Kamendulis LM. Chemical Carcinogenesis. In: Klaassen CD, organizers. Casarett and Doull's Toxicology: The Basic Science of Poisons. New York: McGraw-Hill, 2008. p. 329-79.

43 Lockett KL, Snowhite IV, Hu JJ. Nucleotideexcision repair and prostate cancer risk. Cancer Lett. [Internet] 2005. [cited $2020 \mathrm{Apr}$ 8]; 220(2):125-35. Available from: https:// www.sciencedirect.com/science/article/abs/ pii/S0304383504006688?via\%3Dihub

44 Bhalli JA, Ali T, Asi MR, Khalid ZM, Ceppi MC, Khan QM. DNA damage in Pakistani agricultural workers exposed to mixture of pesticides. Environ Mol Mutagen. [Internet] 2009 Jan. [cited 2020 Apr 19]; 50(1):37-45. Available from: https://onlinelibrary.wiley. com/doi/abs/10.1002/em.20435
45 Adorini L, Penna G, Amuchastegui S, Cossetti C, Aquilano F, Mariani R, et al. Inhibition of prostate growth and inflammation by the vitamin D receptor agonist BXL-628 (elocalcitol). J Steroid Biochem Mol Biol. [Internet] 2007. [cited 2020 Mar 27]; 103:689-93. Available from: https://www.sciencedirect.com/science/ article/abs/pii/S0960076006004420?via\%3Dihub

46 Usmani KA, Cho TM, Rose RL, Hodgson E. Inhibition of the human liver microsomal and human cytochrome P450 1A2 and 3A4 metabolism of estradiol by deployment-related and other chemicals. Drug Metab Dispos. [Internet] 2006. [cited 2020 Apr 8]; 34(9):1606-14. Available from: http://dmd.aspetjournals.org/ content/34/9/1606.long

47 Janssens JP, Van Hecke E, Geys H, Bruckers L, Renard D, Molenberghs G. Pesticides and mortality from hormone-dependent cancers. Eur. J. Cancer Prev. 2001; 10(5):459-67. doi: 10.1097/00008469-200110000-00010.

48 Sawada N, Iwasaki M, Inoue $\mathrm{M}$, Itoh $\mathrm{H}$, Sesazuki S, Yamaji T, et al. Plasma organochlorines and subsequent risk of prostate cancer in Japanese men: A nested case-control study. Environmental Health Perspectives. [Internet] 2010 May. [cited 2020 Apr 02]; 118:659-65. Available from: https://www.ncbi.nlm.nih. gov/pmc/articles/PMC2866682/

49 Carneiro FF, Riggoto RM, Augusto LGS, Friedrich K, Búrigo AC, organizadores. Dossiê ABRASCO: Um alerta sobre os impactos dos agrotóxicos na saúde. Rio de Janeiro: ABRASCO; 2015. p. 628.

50 Belo MSSP, Pignati W, Dores EFGC, Moreira JC, Peres F. Uso de agrotóxicos na produção de soja do Estado do Mato Grosso: um estudo preliminar de riscos ocupacionais e ambientais. Rev Bras Saúde Ocup. [Internet] 2012. [citado 2020 fev 12]; 37(125):78-88. Disponível em: https://www.scielo.br/scielo.php?pid=S030376572012000100011\&script=sci_arttext

Recebido em: 26 fev. 2021 Aceito em: 21 set. 2021 